\title{
Pipeline Development of Skilled Students in Advanced Control Systems
}

\section{Dr. Ahmed Cherif Megri, North Carolina A\&T State University}

Dr. Ahmed C. Megri is an Associate Professor of engineering. He received his HDR (Dr. Habilitation) in Engineering Sciences, from Marie and Pierre Curie University, Paris VI (Sorbonne Universities), in 2011, and his Ph.D. in Thermal Engineering, from Lyon Institute of Technology in 1995. He wrote more than 100 papers in the journal and international conferences. His research interests include thermal and mechanical modeling and simulation of materials. He participates in multiple projects, including the Development of a Model for The Metal Laser Powder Bed Fusion Additive Manufacturing Process. Dr. Ahmed Cherif Megri was the chair of the NCAT CAM's Education subcommittee. He contributed to the outreach CAM since 2015. He is currently, responsible for the outreach program for the STEAM's research project.

Dr. Sameer Hamoush P.E., North Carolina A\&T State University

Professor and Chair of Civil and Architectural Engineering Department 


\title{
Pipeline Development of Skilled Students in Advanced Control Systems
}

\author{
Dr. Ahmed Cherif Megri \& Dr. Sameer Hamoush
}

\begin{abstract}
:
The building automation system (BAS) is part of the University HVAC system. It is used to monitor and save energy through energy performance contracting with different control systems. Recently, the HVAC laboratory facilities (Split-System Central Unit Heat Pump, Air Handling Units (AHUs), Variable Air Volumes (VAVs) box, and even a Wall-Mounted Heat Pump) have been incorporated into the University's BAS system to become a part of students' education.

BAS is the foundation of the energy management efficiency of modern buildings. Basically, a central system, the intelligent Metasys BAS software, connects the HVAC, lighting, security and protection systems and allows them to communicate on a single platform to provide the necessary information to the energy manager; enabling them to make more informed decisions while improving occupant comfort, safety and productivity.

Associated with HVAC systems, several BAS-related peripherals, such as Network Automation Engines (NAE), Input / Output Modules (IOMs), Controllers, Network Control Engines, TEC Thermostat Controllers, the variable air volume assemblies (VMAs), are part of the HVAC system to execute the decision made by the manager, as well as to provide and collect information on the basic function of the systems.

The purpose of this article is to evaluate student learning at the laboratory course level and to determine the extent to which BAS serves students' learning experience in relation to fundamental concepts. More importantly, the methodology related to the BAS project will be discussed. We discuss the project from students' perspective and experience earned in the areas of design, integration. The methodology used to evaluate the effectiveness of this class in terms of learning outcomes is also described.
\end{abstract}

\section{Building Automation:}

The main objective of a Building Automation System (BAS) is to acquire the complete autonomous control of an entire building. Basically, a BAS is designed to monitor and control the mechanical, security, fire, lighting, HVAC and humidity control and ventilation systems in a building or across several buildings [1] to [5].

The BAS is composed of electronic devices and a computer networking that is able to keep building indoor conditions within a specified range, light rooms based on an occupancy schedule, monitor performance and device failures in all systems and provide malfunction alarms. An automation system is able to control an HVAC system based on lighting motion sensors or another type of control based on programming. The goal is to achieve energy efficiency, reduce energy 
consumption, as well as reduce building maintenance costs, while improving thermal comfort, safety and occupant productivity.

Typically, they are financed through energy and insurance savings called Performance Contracting, and other savings associated with pro-active maintenance and quick detection of issues. Energy Performance Contracting is a service which provides customers with comprehensive energy conservation measures, such as improving the efficiency of the HVAC equipment, and upgrading fluorescent fixtures to LEDs. It goes along with guarantees that the savings produced by a project will be sufficient to finance the full cost of the project. Currently, NC State Universities are undergoing multiple energy performance contracting with the state.

The BAS makes the building qualified to be often called an intelligent building or "smart building". The robust BACnet is the communication protocol for Building Automation and Control (BAC) networks that leverage the ASHRAE, ANSI, and ISO 16484-5 standard protocol.

The majority of multi-story green buildings are designed to accommodate a BAS for the energy, air and water conservation characteristics. Electrical device demand response is a typical function of a BAS. Scheduling and using the data collected from the sensors are among the most valuable functions of a BAS system. The collected data from each existing unit, such as chillers, boilers, Air Handling Units (AHUs), and VAV boxes, may be treated and optimized for an improved use of energy within a building.

\section{HVAC Systems LAB connected to the University BAS:}

The Utility Department at NCAT is taking charge of the University BAS. All 17 campus buildings are connected to the University BAS. The HVAC Department uses the BAS to monitor building performance, such as temperature and other systems characteristics over the campus, while maintaining thermal comfort $\left(68^{\circ} \mathrm{F}\right.$ to $\left.75^{\circ} \mathrm{F}\right)$. They use BAS to detect systems deficiencies, such as leaks from units or within the systems.

At the Department of Civil, Architectural \& Environmental Engineering (CAEE), indoor environment laboratory (directed by Dr. Megri), multiple HVAC systems, and teaching labs, have been connected to the BAS system of the University. The systems are:

1) Split-System Central Unit Heat Pump (SSHP): Supply flow into an occupied space as a mixture of return air and fresh outdoor air that has been filtered and then conditioned through heating or cooling coils, paired with a quiet and compact outdoor unit. As you likely gathered from its name, a split system means it has components that are located inside and outside the building. This volume of supply air will become entrained with the room air at a known rate in order to achieve occupant comfort by providing sufficient volume flow to satisfy the number of occupants in a given space (figure 1).

2) AHU \& VFD-Axial fan installation: Unit connected to an axial fan, and a VFD (Variable Frequency Drive) that serves to reduce the energy consumption of the fan engine, in response to the reduction of airflow through the duct, by reducing the frequency (figure 2). The airflow reduction is simulated using a rectangular duct damper. 
3) AHU \& VFD-Centrifugal fan installation: Unit connected to a centrifugal fan, and a VFD that serves to reduce the energy consumption of the fan engine, in response to the reduction of the airflow through the duct, by reducing the frequency (figure 3). The airflow reduction is simulated using a circular duct damper.

4) Wall-Mounted Heat Pump (WMHP): A ductless heat pump consists of an indoor unit, which is window-mounted (figure 4).

To be part of the BAS system, multiple transducers, sensors (temperature, relative humidity and $\mathrm{CO}_{2}$ concentration), and actuators to control the position of the dampers have been added to the system.

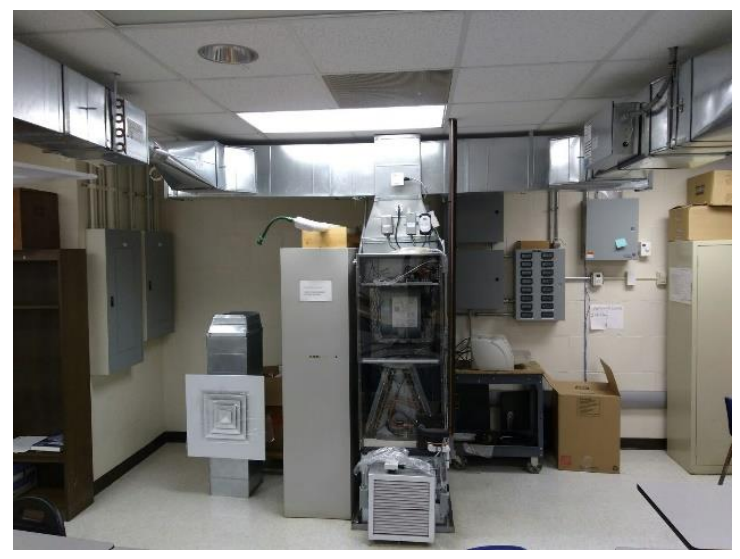

Figure 1: Split-System Central Unit Heat Pump (SSHP)

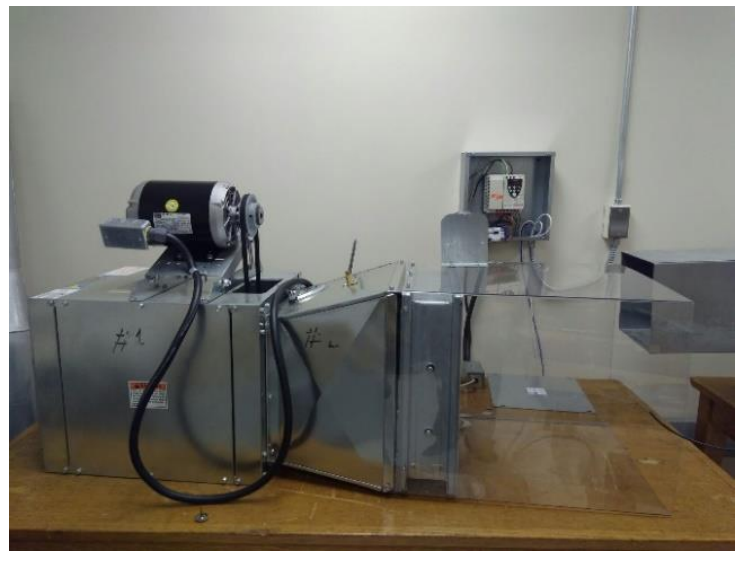

Figure 2: AHU \& VFD-Axial Fan Installation

The main objective of these units and their integration to the BAS is to use them as educational tools to teach HVAC, control systems, visualization of different control components and witness the functions of an integrated system.

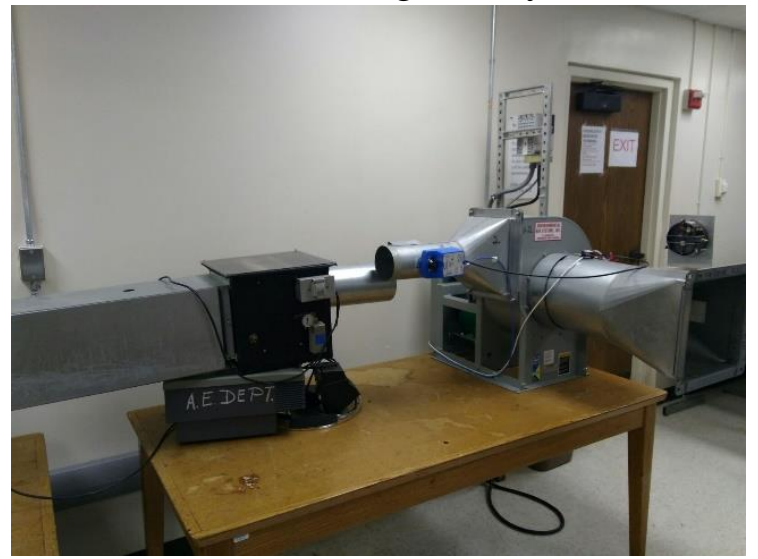

Figure 3: VFD and Centrifugal Fan Installation

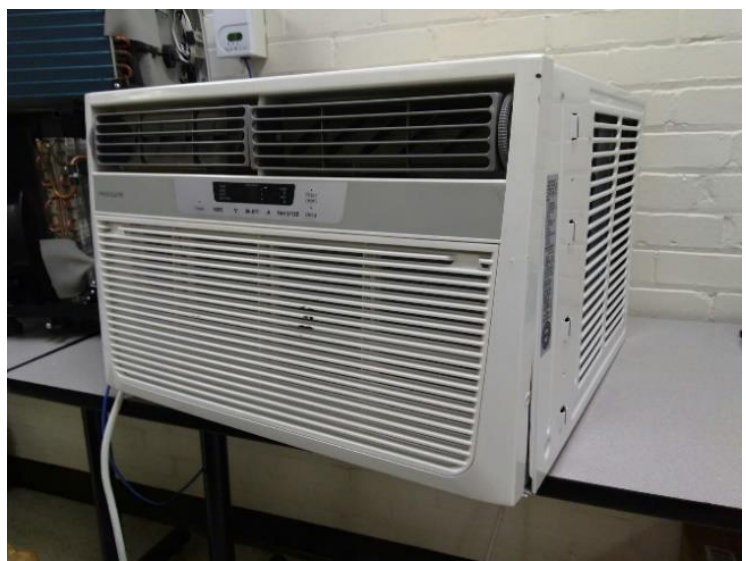

Figure 4: Wall-Mounted Heat Pump (WMHP) 


\section{Sample of the labs activities:}

The four installations connected to the University BAS system are used for the HVAC Lab over one semester. In this labs, the students are experimenting processes, as well as using a building energy simulation program. They measure temperature, airflow, relative humidity, and pressure, as well as $\mathrm{CO}_{2}$ concentration of air at different points of the system: supply, return, mixture, as well as outdoor air. These information are collected either form the computer connected to the BAS system or from a panel board where sensor measurements are displayed instantaneously. These installations were used separately for the HVAC lab. An effort has been made to integrate these installations as a part of the BAS of the University Campus. Currently, these installations can be controlled from a distance using the Metasys launchers. The integration of the LAB HVAC systems, allows the use of the data collected from different sensors located all over the HVAC systems in the campus. The data may be treated, in order to discover patterns in large data sets involving methods based on predictive models using machine learning, statistics, and database systems. Within Matlab, predictive models have been developed, from the data collected, for different HVAC systems: fan, chiller, VAVBox, fan coil units, AHUs, and so on. Predictive models uses data mining and probability to predict results. Each model is composed of a number of predictors, which are variables that can influence future results. Once the data are collected for the relevant predictors, a statistical model is developed. The models were developed by the instructor in collaboration with the students. It is an essential process where intelligent methods are applied to extract data patterns. The information is extracted from a data set and transformed into an understandable structure for further use.

1) Measuring Dry Temperature and Relative Humidity: The purpose of the laboratory exercise is to measure the relative humidity $\mathrm{RH}$ and dry bulb air temperature using various types of thermometer and then find the wet bulb, dew point, specific volume, humidity ratio, and enthalpy of moist air through the psychometric chart. The two installations SplitSystem Central Unit Heat Pump (SSHP) and Wall-Mounted Heat Pump (WMHP) is used for this purpose. Both paper and computer program psychrometric charts are used.

2) Heating Process: The purpose of this laboratory exercise is (1) To measure the airflow rates, relative humidity RHs, and dry bulb air temperatures entering and leaving the heating system; (2) Calculate the heat added to the air stream (btu/hr); and (3) Represent the heating process on the psychrometric chart. This process is repeated for several indoor conditions, controlled using the wall thermostat or the computer program associated with the BAS system.

3) Cooling Process: The purpose of this laboratory exercise is (1) To measure the airflow rates, relative humidity RHs, and the dry bulb air temperatures entering and leaving the cooling system; (2) Calculate the heat and moisture removed from the air stream; and (3) Represent the cooling process on the psychrometric chart. This process is repeated for several indoor conditions, controlled using the wall thermostat or the computer program associated with the BAS system.

4) Adiabatic Mixture: The purpose of this laboratory exercise is to study the adiabatic mixture of two air streams and represent the process on the psychrometric chart. 
5) Building Energy Simulation Software eQuest: The purpose of this laboratory exercise is to (1) Explore the energy simulation software eQuest; and (2) Study the impact of different $\mathrm{U}$ value of the walls on building loads and the annual energy consumption.

6) Window characteristics: The purpose of this laboratory exercise is to measure (1) Solar heat gain coefficient SHGC; (2) Ultraviolet UV; (3) The visibility; and (4) Inside surface temperature of the window, along with studying the impact of different window types on building loads and energy consumption by using the energy simulation software eQuest.

7) Energy Conservation Measures (ECM): The purpose of this laboratory exercise is to (1) Use simulation software to explore the energy use for an existing building that has been modeled by the software; (2) Use different energy conservation measures, investigate the savings obtained from each, and determine the Energy Use Intensity (EUI) for each measure.

\section{Equipment used to measure the airflow diffusers:}

The Exhaust Fan Flow Meter was designed to make quick and accurate measurements of air flow through exhaust fans and diffusers. The effective air flow measurement range for the Exhaust Fan Flow Meter is 10 to $124 \mathrm{cfm}$ (Figure 5).

The TrueFlow Air Handler Flow Meter is designed to provide a simple and accurate measurement of airflow through residential air handlers, similar to the installation available in the teaching lab. The TrueFlow Meter temporarily replaces the filter in the air handler distribution system during the airflow measurement process. The TrueFlow Meter provides direct CFM readings in 2 to 3 minutes without extensive calculations or setup. It is designed to measure flow through air handlers rated from 1 to 5 tons, can be used in a wide range of return plenums and air handler fan configurations, has adjustable sizing to fit standard and custom filter slots, and it is proven to be more accurate than the single-point temperature rise method and easier than the fan curve method (Figure 6).

\section{Digital capture hood measuring a supply diffuser (Measuring Air Velocity and Air Volume at Supply Grilles/Diffusers):}

Capture hoods, rotating vanes, and thermal anemometers are the most common choices used to verify proper minimum flow rates; confirm proper operation of dampers, controls, and fans; and ensure the efficient use of energy (Figure 7).

Capture hoods are popular instruments to use for measuring flow rates from supply grilles. They provide a direct air volume reading, and come with a variety of hood sizes to match various grilles. Thus, capture hoods are often the tool of choice (when measuring supply). 
ASHRAE recommends performing a duct traverse to determine if a correction factor is needed for air capture hood measurements. Different diffuser styles, elbows attached directly to the diffuser, and dampers located just upstream of the diffuser can impair the uniformity of the flow patterns coming out of the diffuser and affect the hood reading.

Utilizing these instruments effectively can help you ensure the proper balance of mechanical HVAC system supply airflows.

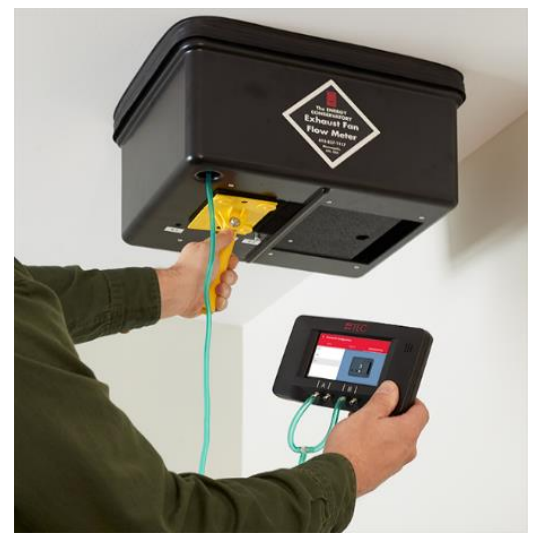

Figure 5: Exhaust Fan Flow Meter [8]

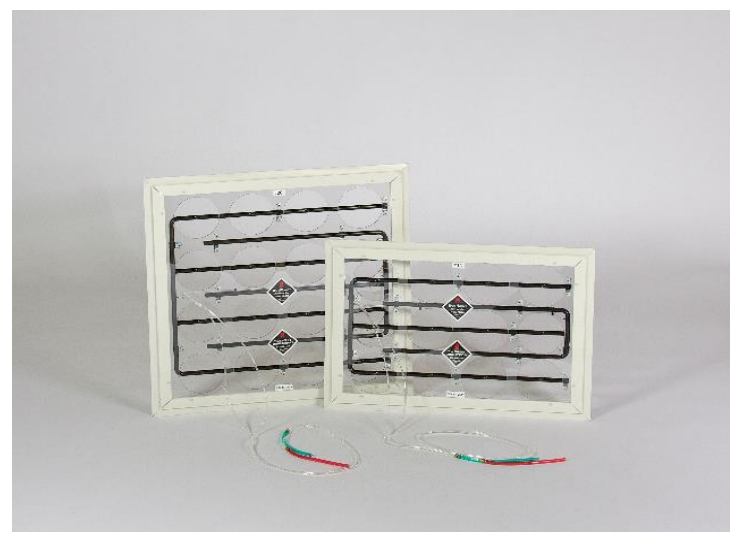

Figure 6: The TrueFlow ${ }^{\circledR}$ Air Handler Flow Meter [8]

\section{Data Mining:}

The Support Vector Machine learning tool has been used to analyze data collected from the BAS of the NCAT University, Academic Classroom Building, AHU 4. The Figure 8 shows the BAS interface, showing an AHU of a specific building.

Scheduling and using the data collected from the sensors are among the most valuable functions of a BAS system. The collected data from each existing unit, such as chillers, boilers, Air Handling Units (AHUs), VAV boxes, may be treated and optimized for an improved use of energy within a building. Two examples of prediction are shown in figures 9 and 10. The figures 9 and 10 show respectively the Supply Air Fan (AHU SAF) as function of the air handling unit chilled water valve (AHU4 CHW VLV), and the supply fan speed (AHU4 SA Speed), taken from the BAS, as well as the prediction model, developed using the support vector machine learning tool [6], [7] that was developed in the frame of a research project, conducted by Dr. Megri. The flow chart of figure 11 shows the process used for our system optimization. 

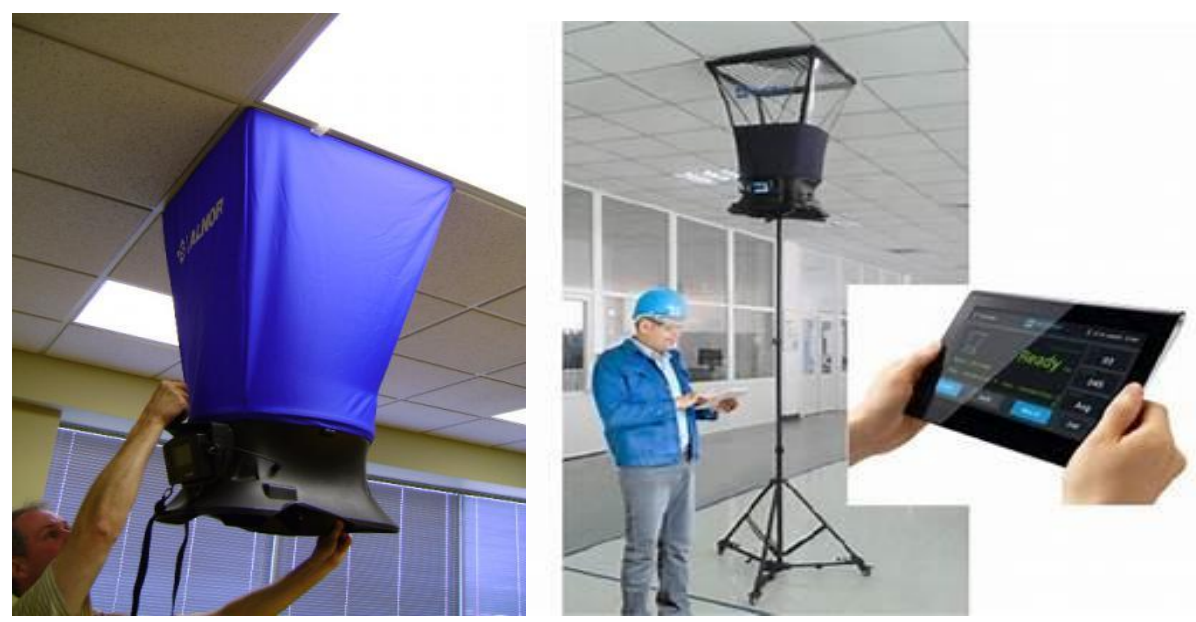

Figure 7: Airflow Capture Hood [9]

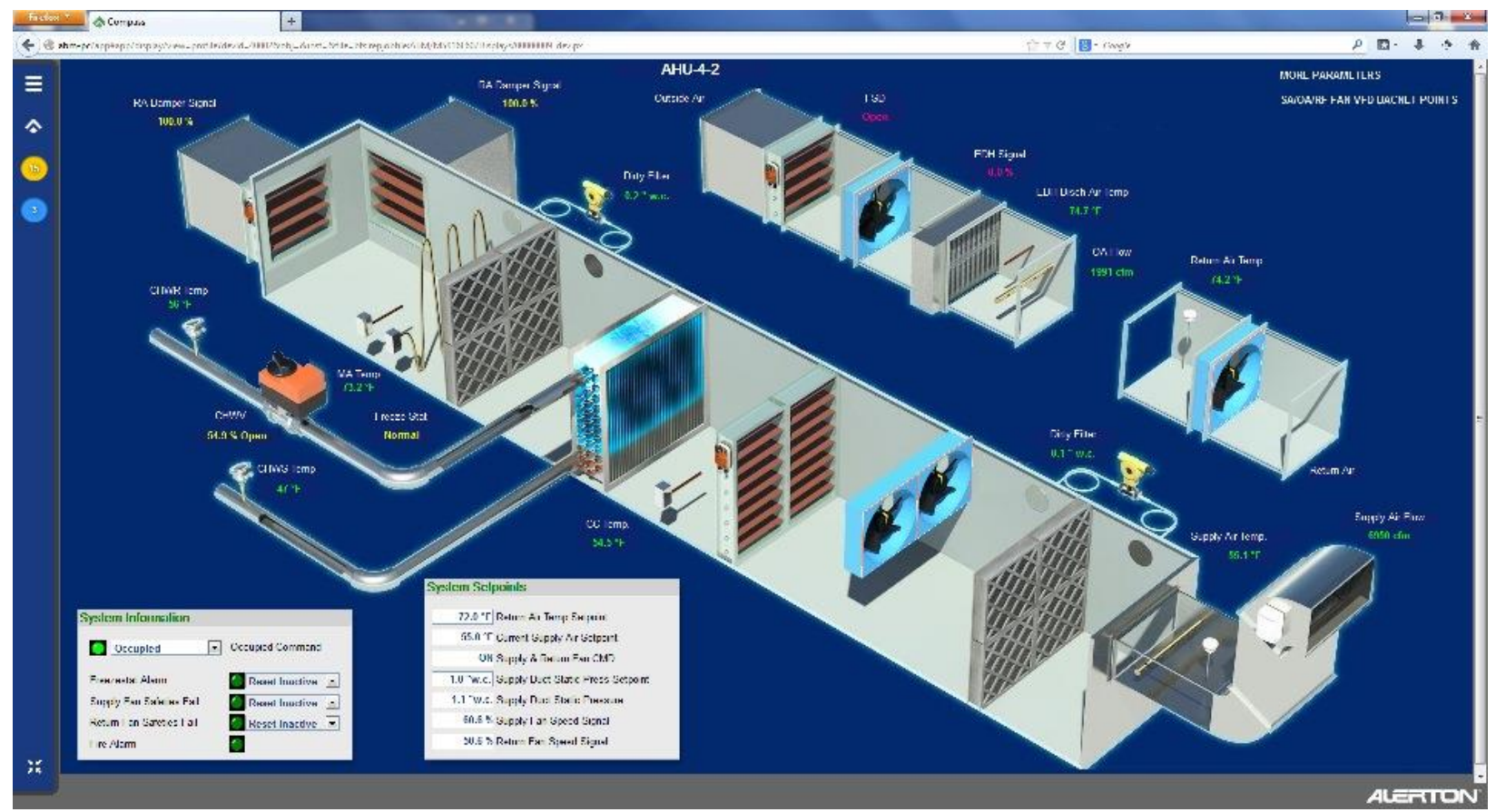

Figure 8: The BAS interface, showing an AHU of a specific building

Many other statistical predictive methods were developed and tested, such as PNN (probabilistic neural network) /GRNN (general regression neural network) Neutral Network, GMDH (Group Method of Data Handling) polynomial network, and Decision Tree Forest. These techniques may be useful for comparison and evaluation purposes. 


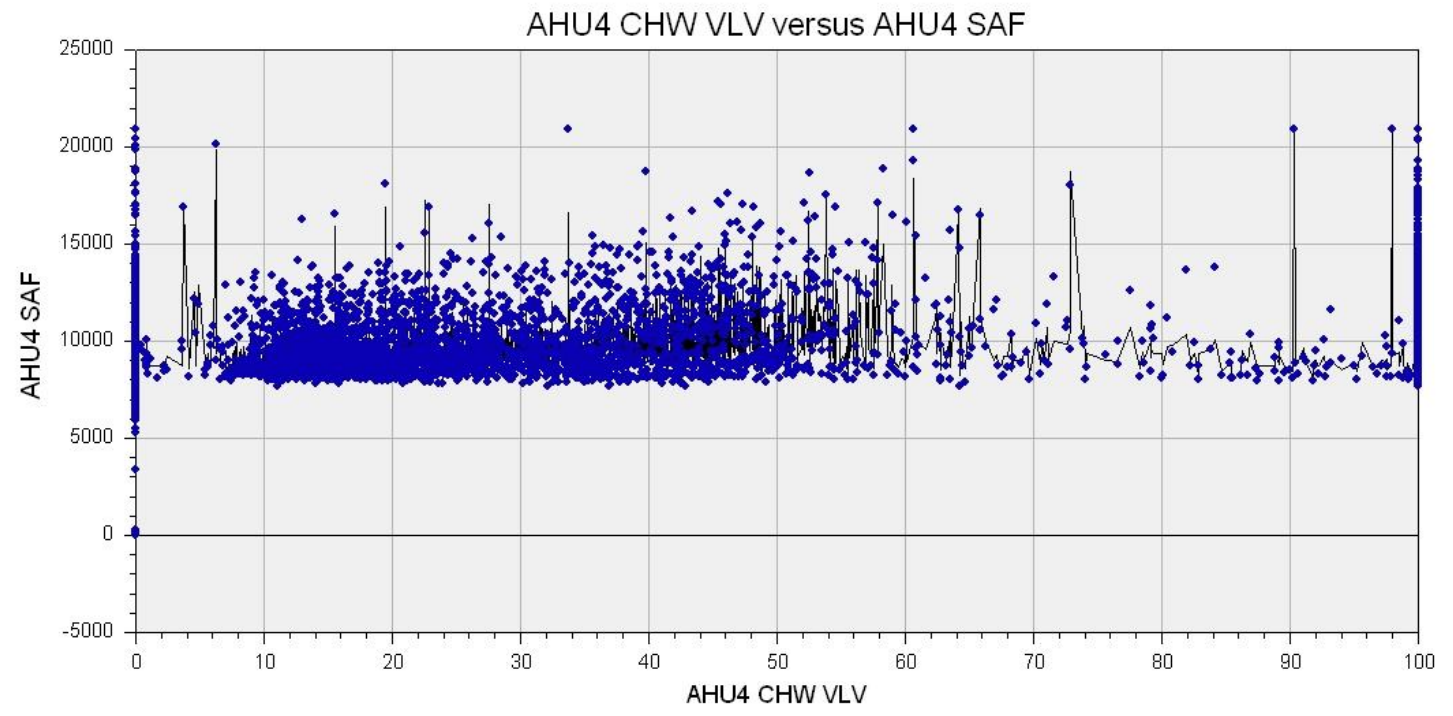

Figure 9: Data Mining, using Support Vector Machine learning tool. AHU SAF (Supply Air Fan) as a function of CHW VLV (Chiller Valve), taken from BAS

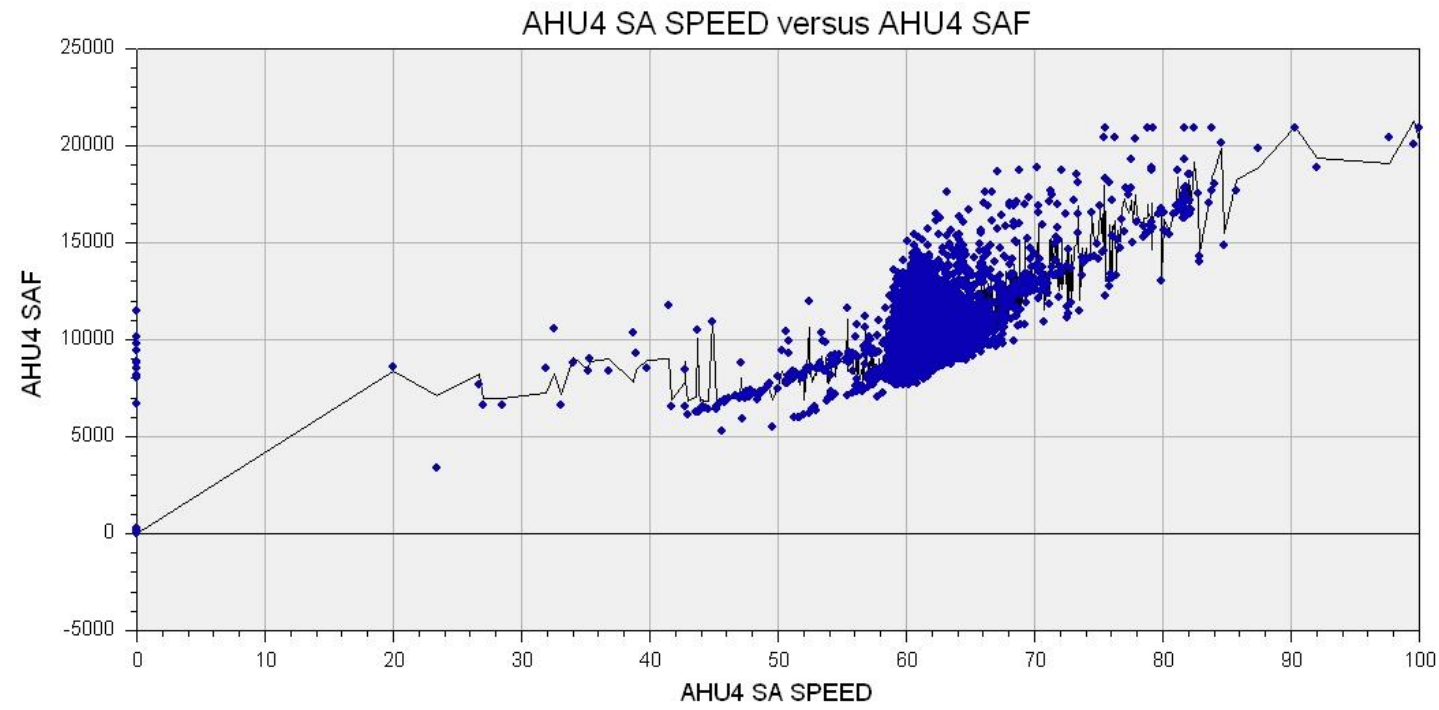

Figure 10: Data Mining, using Support Vector Machine learning tool. AHU SAF (Supply Air Fan) as a function of Supply Air Speed, taken from BAS

The consequence of the use of BAS is a large amount of data. These data represent the behavior of each system, such as a fan, chiller, boiler, etc. Figures 9, 10 and 11 show how this data can be collected, used to form the predictive models, then to predict different parameters related to these systems. 


\section{Evaluation and Methodology:}

This evaluation research utilizes a mixed-methods approach employing both qualitative and quantitative data sources to determine the impact of the HVAC Labs, using BAS system on student learning. Mixed methods designs are methodologically superior to simpler designs because of the ability to leverage the strengths of several different methods. Consistent data from both qualitative and quantitative methods increases the trust worthiness of the findings.

Using the indirect course evaluation form, students were asked, anonymously, to self-assess their ability in specific areas identified by the instructor in connection with the course learning objectives, as well as the motivations for the program experience.

- More Understanding of Data Mining, after the lab classes

- How good was your understanding of the BAS after the HVAC Labs

- How BAS labs helped you to understand better the HVAC topics

- Does BAS make building more efficient

- The introduction of BAS seems to make you understand the HVAC subject better

- Does BAS change your mind to select the HVAC concentration

- Does BAS make the labs more interesting

- Does going through the HVAC labs make you more interested to know more about Data Mining

The compilation of the results of the student self-assessment of course learning objectives questions for this short course are presented in Table 1. The student responses of "5" (Extremely Agree) through "1" (Disagree).

The results of the students' assessment show that for all the questions, students generally feel like BAS helps them to understand better the HVAC topics, and are interested to know more about Data Mining. The assignments and performance of the students over the labs show the same positive answers.

The instructor also conducted an evaluation of the performance of students in the course as part of the Program Objectives (PO) and outcome assessment process. A summary report on the performance of students (to meet the program objectives) and compliance with the program outcomes is prepared and submitted to the funding agency (DOE). A more rigorous process in assessing the learning outcomes of this lab course will be implemented, which is in parallel with the program outcomes. The following outlines process will be used for this course assessment.

- Individual instructor evaluation of the degree of learning achievement of individual students on a team, which includes consideration of the collective achievements of the team.

- Peer evaluation (optional by instructor).

- Grading of deliverables by the instructors (projects evaluation, oral presentation, team minutes, and website if applicable).

- Teamwork survey

- Self-assessment 


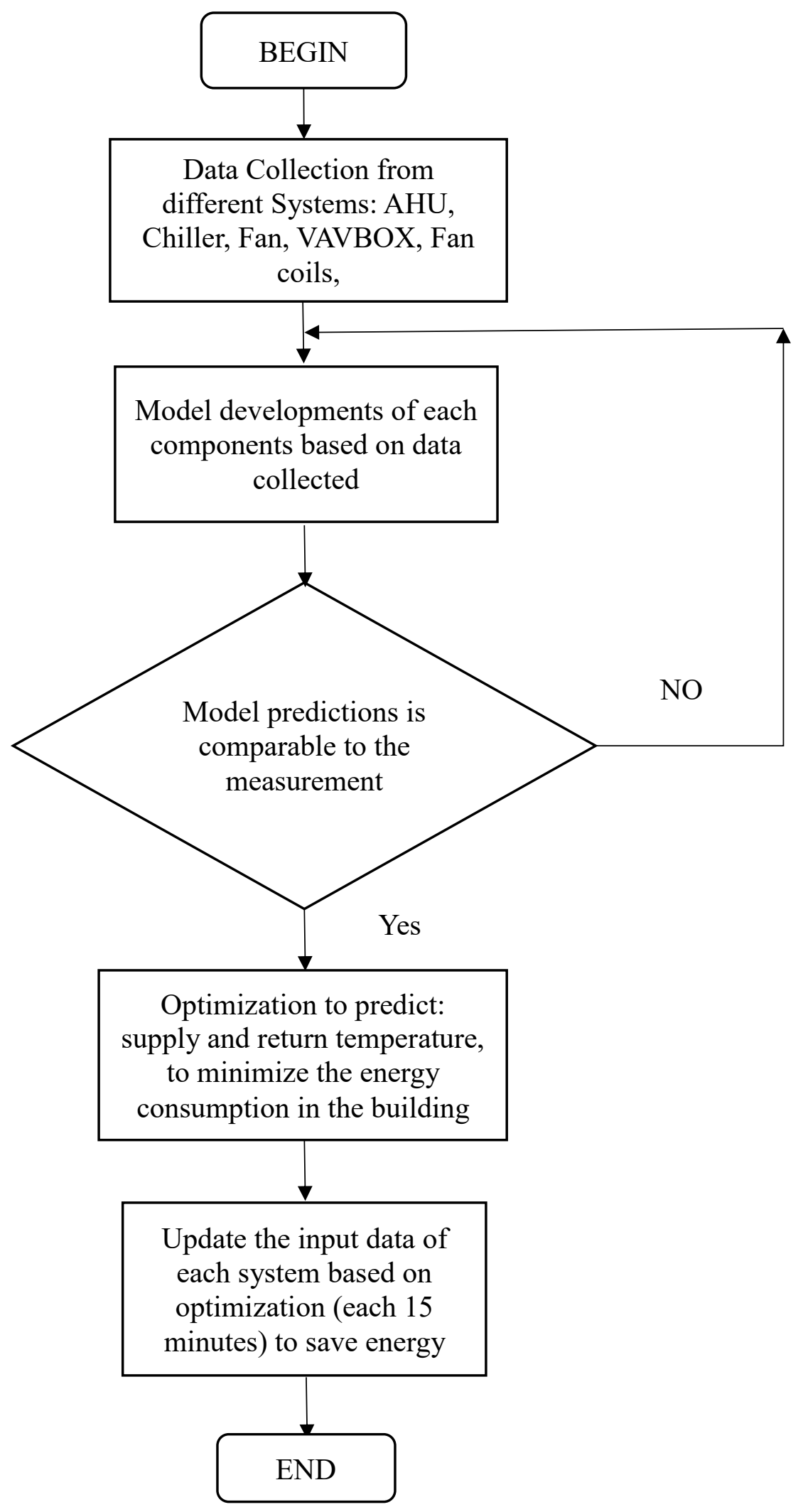

Figure 11: Flow chart, used for the optimization process. 
Table 1: Indirect Assessment

\begin{tabular}{|c|c|c|c|c|c|c|}
\hline \multicolumn{7}{|c|}{ Indirect Assessment (14 students) } \\
\hline $\begin{array}{l}\text { Student Self-Assessment of } \\
\text { Course Learning Objectives }\end{array}$ & $\begin{array}{l}\text { Extremely } \\
\text { Agree (5) }\end{array}$ & $\begin{array}{l}\text { Strongly } \\
\text { Agree (4) }\end{array}$ & Agree (3) & $\begin{array}{l}\text { Somewhat } \\
\text { Agree (2) }\end{array}$ & $\begin{array}{l}\text { Disagree } \\
\text { (1) }\end{array}$ & Equivalent \\
\hline $\begin{array}{l}\text { More Understanding of Data } \\
\text { Mining, after the lab classes }\end{array}$ & 6 & 6 & 1 & 1 & & 3.93 \\
\hline $\begin{array}{l}\text { How good your understanding } \\
\text { of the BAS after the HVAC } \\
\text { Labs }\end{array}$ & 5 & 6 & 2 & 1 & & 3.80 \\
\hline $\begin{array}{l}\text { How BAS labs helped you to } \\
\text { understand better the HVAC } \\
\text { topics }\end{array}$ & 8 & 2 & 2 & 2 & & 3.87 \\
\hline $\begin{array}{l}\text { Does BAS make building more } \\
\text { efficient }\end{array}$ & 7 & 3 & 2 & 2 & & 3.80 \\
\hline $\begin{array}{l}\text { The introduction of BAS } \\
\text { seems to make you understand } \\
\text { the HVAC subject better }\end{array}$ & 8 & 2 & 2 & 2 & & 3.87 \\
\hline $\begin{array}{l}\text { Does BAS change your mind } \\
\text { to select the HVAC } \\
\text { concentration }\end{array}$ & 4 & 4 & 6 & & & 3.60 \\
\hline $\begin{array}{l}\text { Does BAS make the labs more } \\
\text { interesting }\end{array}$ & 7 & 1 & 5 & 1 & & 3.73 \\
\hline $\begin{array}{l}\text { Does going through the HVAC } \\
\text { labs make you more interested } \\
\text { to know more about Data } \\
\text { Mining }\end{array}$ & 4 & 4 & 4 & 2 & & 3.47 \\
\hline
\end{tabular}

In another record, students taking this lab were successful twice during the last three years in ASHRAE Design competition, where they were ranked second and third.

\section{Conclusions:}

Teaching the HVAC and Electrical Labs is one of the characteristics of our Architectural Engineering Curriculum. We believe that the labs help students to better understand the fundamentals and the principles taught in the classroom. Recently, we integrated the HVAC lab, as well as part of the electrical lab to be part of the BAS system of the University. Our objective is to take advantage of what the BAS offers, from monitoring, control, data mining, data treatment and analysis. This effort has been supported by major companies. Our intent is to use such system to improve the students' understanding of HVAC, control systems, as well as smart buildings and building automation systems.

The survey taken at the end of the HVAC lab class encourages us to continue with other future integrations, such as lighting control, and integration lighting-HVAC system, for better energy performance.

Another objective is to use these installations to teach retro commissioning, where the purpose is commissioning the existing buildings, to improve how building equipment and systems function 
together. Depending on the state of the building, retro commissioning may usually solve problems occurred during design or construction, or address problems that have developed throughout the building's service. The objective is to improve the building operation and maintenance in order to enhance the building performance.

Other HVAC systems, such as a comprehensive movable Hydronic system with two Heat Pumps, a tank, and multiple temperature, pressure and water flow measurement sensors will be connected to the BAS University system, to serve for education purposes.

\section{References:}

[1] Makarechi, S. (2004). Building Automation Systems at the Crossroads. Consulting Specifying Engineer: 4.

[2] Makarechi, S. (2005). A Step Towards Development of Performance Indicators for Building Automation Systems. Georgia Tech: 38.

[3] Bowen, T. S. (2005). "Overly Smart Buildings." Technology Research News. BuilConn (2004).

[4] Converging Building Systems Technologies. BuilSpec Educational Seminar 2004, Atlanta, GA, BuilConn.

[5] GSA (2005). General Services/Energy \& Building Automation. Annual Performance Measurement Report. GSA. Washington DC, GSA: 4.

[6] "Data Mining Curriculum". ACM SIGKDD. 2006-04-30. Retrieved 2014-01-27.

[7] Jiawei Han, Micheline Kamber, Jian Pei (June 9, 2011). Data Mining: Concepts and Techniques (3rd ed.). Morgan Kaufmann. ISBN 978-0-12-381479-1.

[8] https://energyconservatory.com/products/product/trueflow/

[9] http://flowmeters.sg/balometer-flow-hood.html

\section{$\underline{\text { Acknowledgement }}$}

This research was supported by DOE, Award Number DE-NA0003867. This work would not have been possible without the support of DOE. 